\title{
Influence of temperature and vacuum on nano- diamond filed emission
}

\author{
Yanning Yang, Zhiyong Zhang and Jiangni Yun / 1st \\ Institute of Photonics \& Photo-Technology and \\ Provincial Key Laboratory of Photoelectronic \\ Technology, Northwest University, Xi'an, China \\ yayyn@163.com
}

\author{
Yanning Yang, Weihu Zhang and Fuchun Zhang / 2nd \\ College of Physics \& Electronic Information, Yanan \\ University, Yan'an, China
}

\begin{abstract}
In order to obtain the stabilized operating temperature and vacuum of nano-diamond filed emission display, the influence of temperature and vacuum changes on nano-diamond filed emission was studied, and the mechanism of field emission under high temperature and low vacuum was analyzed. It was found that nano-diamond filed emission turn-on filed decreases, and current density increases as the temperature increases. After the temperature increases to $130{ }^{\circ} \mathrm{C}$, filed emission starts to deviate from the traditional Fowler-Nordheim theory. Under low vacuum, nano-diamond field emission is very unstable. As the vacuum increases, turn-on filed decreases, and current density increases. However, when the vacuum increases to $10^{-4} \mathrm{~Pa}$ level, turn-on filed, current density, and luminescence effect becomes stable. vacuum can decrease with the increase of temperature. When the temperature is below $150{ }^{\circ} \mathrm{C}$, the decrease of the vacuum will not influence the stability of field emission. When the system operates stably, the temperature of cathode surface should be below $130^{\circ} \mathrm{C}$, and the vacuum should be above $10^{-4} \mathrm{~Pa}$ level.
\end{abstract}

Keywords-temperature; vacuum; nano-diamond; field emission

\section{INTRODUCTION}

Diamond is a special kind of semiconductor material with good properties, ${ }^{[1]}$ such as high hardness, broad-band gap, high heat conductivity, thermal stability, resistance to chemical attack. Meanwhile, with negative electron affinity, diamond films have low work function. Emitter, made of semiconductor materials, has very bad heat dissipation when operating at high current. ${ }^{[2]}$ However, the high heat conductivity of diamond ensures a good heat dissipation of diamond field emission display (FED), so it is generally believed that diamond is a kind of excellent field electron emission material. On the other hand, semiconductor emitter, easy to be oxidized, has an influence on the stability of electron emission. Because of the chemical activity of semiconductor, the remaining particles adsorb and are desorbed easily on the surface of semiconductor so that emission stability is influenced. Therefore, semiconductor field emission devices should operate in the higher vacuum. Some researches indicate that activity and size effect of nano particle has certain influence on field emission. ${ }^{[3]}$ Besides, the traditional Fowler-Nordheim field emission theory is put forward for metal material, so diamond field emission does not follow this theory fully, and the mechanism of diamond field emission has yet to be revealed wholly. Especially there is less study on the influence of temperature and vacuum on filed emission. Studying the influence of temperature and vacuum on filed emission characteristics is favorable for exploring the mechanism of nano-diamond electron emission, for determining its limit operating parameter, and for providing the basis to the design and manufacture of nano-diamond field emission display.

\section{EXPERIMENT}

The test system, placed in vacuum chamber, mainly consists of diode structure FED cathode sample, anode, temperature thermocouple, vacuum gauge, tungsten lamp, applied excitation power supply, etc. Firstly, using vacuumpumping system keeps the furnace vacuum at a point within $10^{-}$ ${ }^{4} \mathrm{~Pa}$ level. Then, heating the sample in the furnace by tungsten lamp makes the temperature of sample surface rise gradually from $20^{\circ} \mathrm{C}$ to $170^{\circ} \mathrm{C}$. In the process, to measure field emission current of the sample, each measurement should be done after one minute when the temperature reaches at sampling point. The experiment of the influence of vacuum on field emission will test the field emission characteristics of nano-diamond cathode sample when the furnace vacuum is in the range from $10^{-1} \mathrm{~Pa}$ level to $10^{-5} \mathrm{~Pa}$ level.

\section{RESULTS AND ANALYSIS}

\section{A. Influence of temperature on field emission}

1) Influence of temperature on field emission turn-on filed: Figure 1 is relation curve between turn-on field of typical sample and temperature. It can be observed that as the increase of temperature beginning with $20{ }^{\circ} \mathrm{C}$, the field emission turn-on filed shows a downward trend. The higher the temperature is, the lower the field emission turn-on filed will be.

The project was supported by the National Natural Science Foundation (60976069) and the Special Foundation of Education Department of Shaanxi Province $(08 \mathrm{jk} 487)$. 


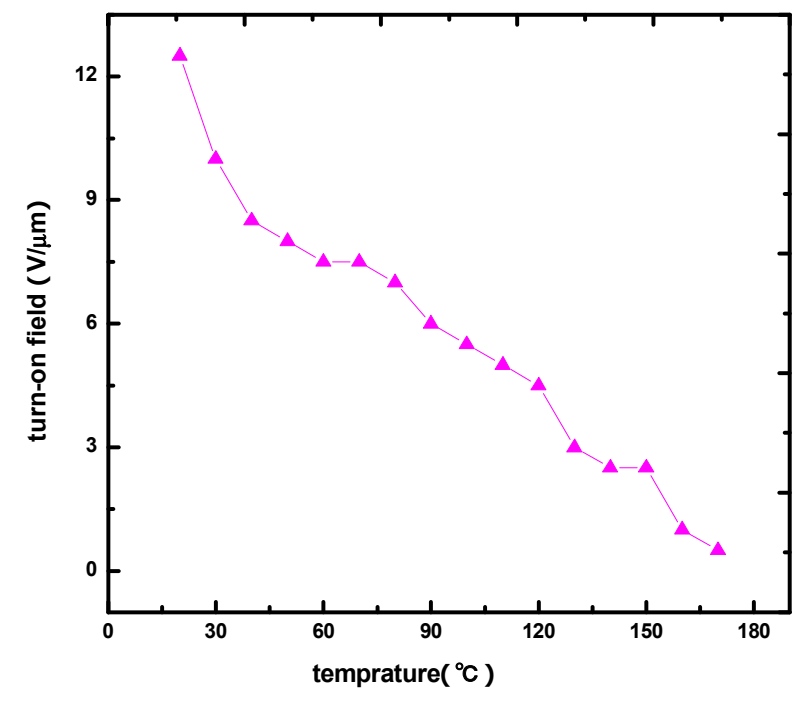

Figure 1. Relation curve between turn-on field of typical sample and temperature

2) Influence of temperature on field emission current density: Figure 2 is J-E characteristic curve of typical sample at different temperatures. It can be seen that with the increase of temperature, field emission current density presents an upward trend. The higher the temperature is, the larger the field emission current density will be. ${ }^{[4]}$

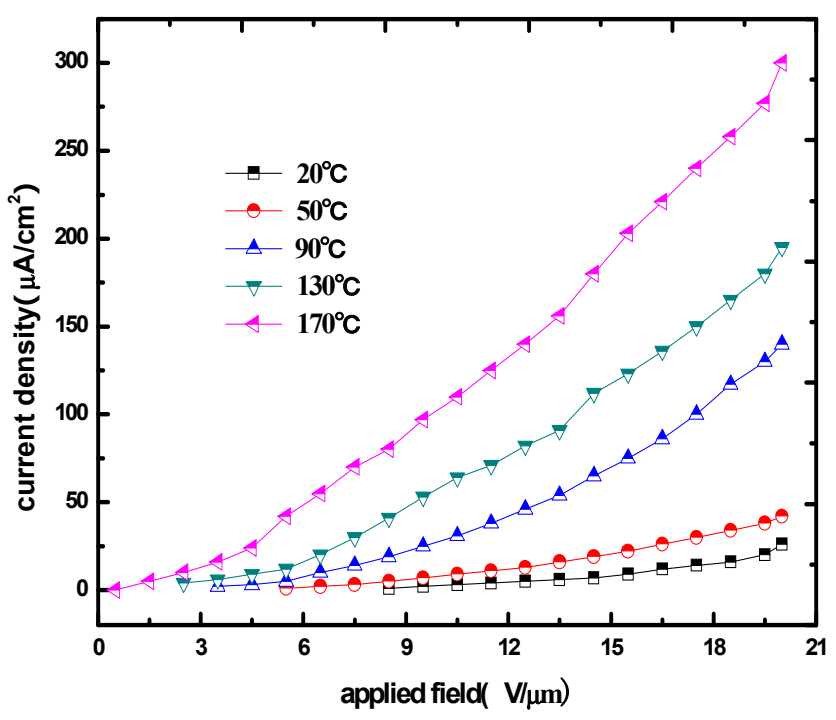

Figure 2. J-E characteristics curve of typical sample at different temperatures

3) F-N curve of tipical sample at different temperatures : Figure 3 is F-N curve of tipical sample at different temperatures. At low temperatures, with the field emission current of the sample as the dominance, the curve is approximately a straight line, which conforms to the F-N theory. As the increase of temperature, the slope of F-N curve becomes smaller, the steepness reduces, and the field emission characteristics are improved. After the temperature increases to $130^{\circ} \mathrm{C}, \mathrm{F}-\mathrm{N}$ curve starts to rise and gradually deviates from the theory of field emission. Furthermore, the higher the temperature is, the more the deviation will be.

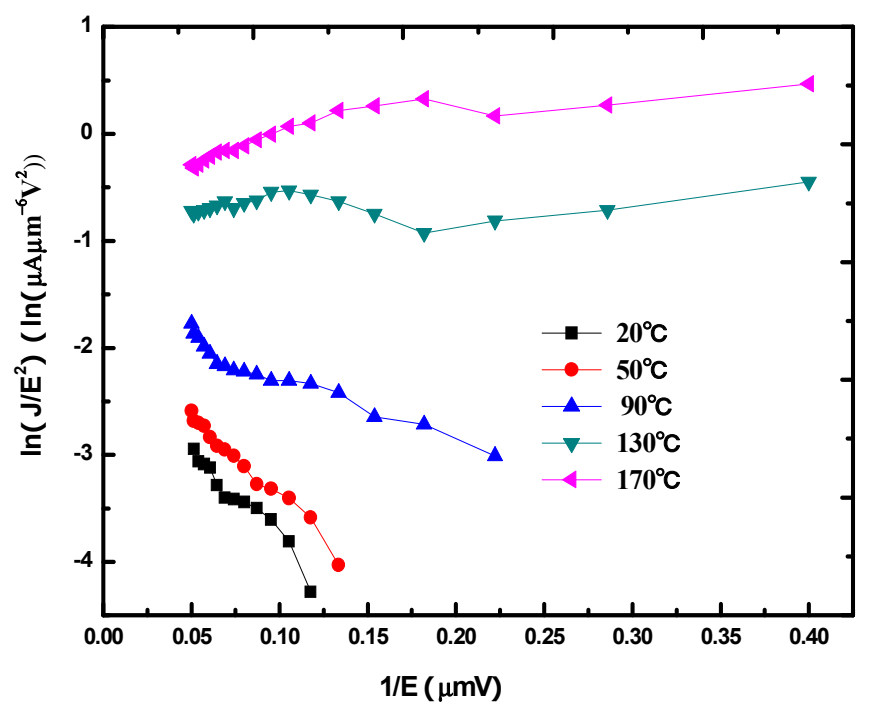

Figure 3. F-N curve of the typical sample at different temperatures

Due to the influence of size effect, thermal conductivity of nano-diamond particles is lower than the one of bulk diamond. Field emission cathode adopts nano-diamond as its electron emitter, and the approach of its heat dissipation is heat exchange. Due to size effect, in applied field, the heat dissipation of the particle surface becomes slow, and its temperature increases as its thermal conductivity reduces. The sum of the temperature produced by field emission current of diamond surface and the temperature applied makes the temperature of emitter tip far above the environmental temperature. ${ }^{[5]}$ With the rise of the temperature and the electron energy to a certain extent, the phenomenon of thermal electron emission, represented by the deviation of F-N curve from theory curve, will be generated.

\section{B. Influence of vacuum on field emission}

1) Influence of vacuum on turn-on field: Experiments show that field emission turn-on field is particularly high in the vacuum of $10^{-1} \mathrm{~Pa}$ level, and the ammeter pointer shakes strongly with large swing amplitude, unreadable. Because of more gas molecule in field emission furnace collides with electron and scatters, which makes the current unstable. Turnon field is still relatively large in the vacuum of $10^{-2} \mathrm{~Pa}$ level, and the ammeter hand still shakes. The big reading error means little to data analysis, so the test and record will start with the vacuum of $10^{-3} \mathrm{~Pa}$ level. Figure 4 is relation curve between the experimental vacuum and turn-on field. 


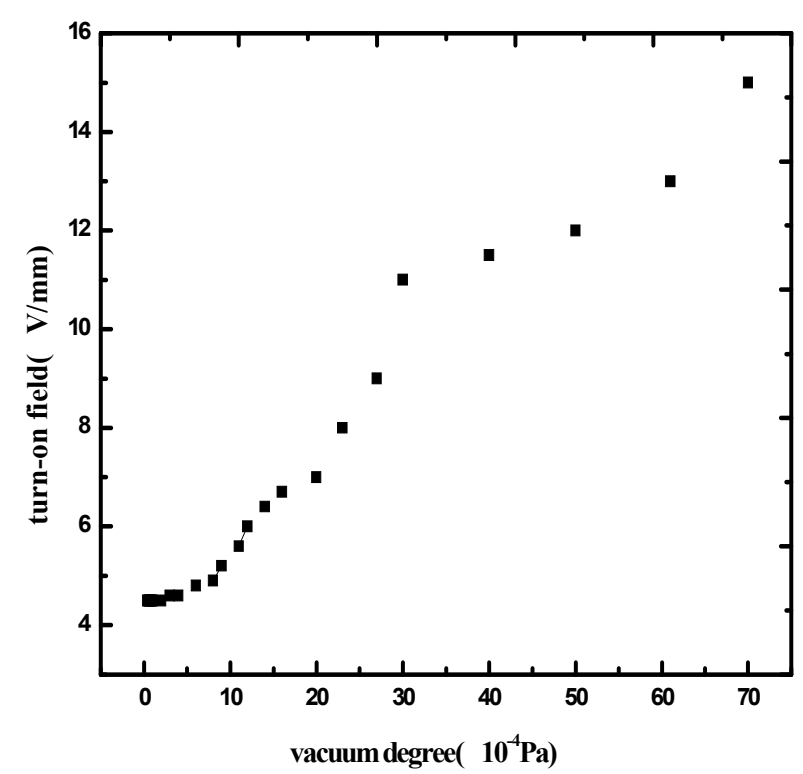

Figure 4. Relation curve between the experimental vacuum and turn-on field

In lower vacuum, field emission turn-on field is higher. As the rise of vacuum degree, turn-on field reduces gradually. Turn-on field will reach up to $15 \mathrm{~V} / \mu \mathrm{m}$ in the vacuum of $7.0 \times 10^{-3} \mathrm{~Pa}$; Turn-on field is $11 \mathrm{~V} / \mu \mathrm{m}$ under the vacuum of $3.0 \times 10^{-3} \mathrm{~Pa}$; Turn-on field reduces to $6.0 \mathrm{~V} / \mu \mathrm{m}$ when the vacuum is $1.3 \times 10^{-3} \mathrm{~Pa}$. After the furnace vacuum rises to $10^{-}$ ${ }^{4} \mathrm{~Pa}$ level, turn-on field becomes stable. Turn-on field is still $6.0 \mathrm{~V} / \mu \mathrm{m}$ under the vacuum of $4.6 \times 10^{-4} \mathrm{~Pa}$. The test turn-on fields are all $4.5 \mathrm{~V} / \mu \mathrm{m}$ in the vacuum from $2.0 \times 10^{-4} \mathrm{~Pa}$ to $5.0 \times 10^{-5} \mathrm{~Pa}$. the lower and stable turn-on field will be obtained instantly as long as the vacuum rises to $10^{-4} \mathrm{~Pa}$ level.

2) Influence of vacuum on current density:Under different vacuum, the current density will be tested in the applied field of $20 \mathrm{~V} / \mu \mathrm{m}$. The relation between system vacuum and current density is shown as in the Figure 5 . In the vacuum from $7 \times 10^{-}$ ${ }^{3} \mathrm{~Pa}$ to $3 \times 10^{-3} \mathrm{~Pa}$, current density has little change about $15 \sim$ $35 \mu \mathrm{A} / \mathrm{cm}^{2}$. In the vacuum from $3 \times 10^{-3} \mathrm{~Pa}$ to $1.1 \times 10^{-3} \mathrm{~Pa}$, as the rise of vacuum, current density will increase quickly from $35 \mu \mathrm{A} / \mathrm{cm}^{2}$ to $117 \mu \mathrm{A} / \mathrm{cm}^{2}$. When the vacuum is close to $10^{-4} \mathrm{~Pa}$ level, field emission current becomes stable gradually. Respectively, the measured current density, which has already been stable, is $117 \mu \mathrm{A} / \mathrm{cm}^{2}, 118 \mu \mathrm{A} / \mathrm{cm}^{2}, 119 \mu \mathrm{A} / \mathrm{cm}^{2}$, and $118 \mu \mathrm{A} / \mathrm{cm}^{2}$ in the vacuum of $1.1 \times 10^{-3} \mathrm{~Pa}, 5.0 \times 10^{-4} \mathrm{~Pa}, 3.0 \times 10^{-}$ ${ }^{4} \mathrm{~Pa}$, and $5.0 \times 10^{-5} \mathrm{~Pa}$.

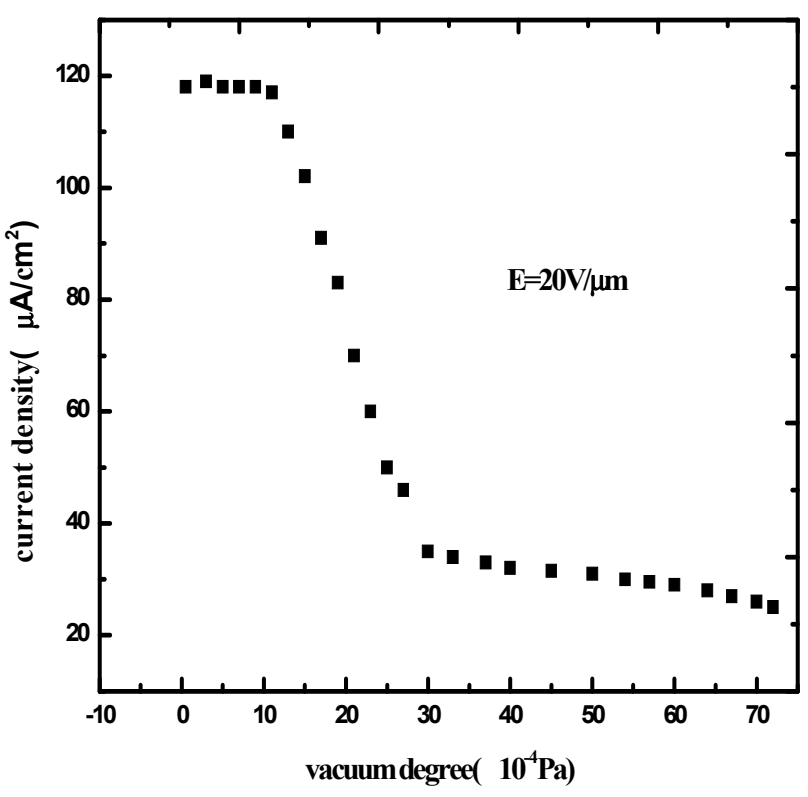

Figure 5. Relation between system vacuum and current density

3) Influence of vacuum on luminescence effect:Figure 6 is the anode luminescence pictures of the typical sample in different vacuum when the applied field is $20 \mathrm{~V} / \mu \mathrm{m}$. As shown here, in the vacuum of $3.8 \times 10^{-3} \mathrm{~Pa}$, the luminescence picture is darker with less luminous point. However, in the vacuum of $8.4 \times 10^{-4} \mathrm{~Pa}, 1.5 \times 10^{-4} \mathrm{~Pa}$, and $5.0 \times 10^{-5} \mathrm{~Pa}$, these luminescence pictures are lighter with more luminous point, which indicates that after the vacuum reaches to $10^{-4} \mathrm{~Pa}$ level, the amount and the illumination of luminous point has already reached a better level. The further increase of vacuum has little improvement on luminescence effect.

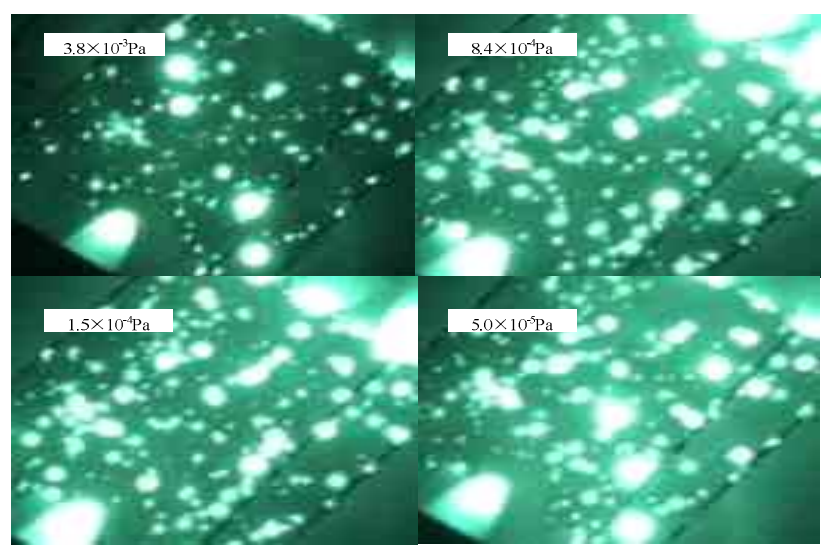

Figure 6. The anode luminescence pictures of the typical sample in different vacuum $(\mathrm{E}=20 \mathrm{~V} / \mu \mathrm{m})$ 


\section{Influence of temperature on vacuum}

It is found that furnace temperature has an influence on vacuum when the experiment of the influence of temperature on field emission is done. Figure 7 is the relation curve between vacuum and surface temperature of vacuum furnace heated after the vacuum of field emission test system reaching to $5.0 \times 10^{-5} \mathrm{~Pa}$ level. As the increase of temperature, the vacuum will decrease gradually. When the diamond coating temperature rises to $150^{\circ} \mathrm{C}$, the system vacuum will decrease to $10 \times 10^{-4} \mathrm{~Pa}$. This is because the gas molecule adsorbed on the inner wall of vacuum chamber and desorbed with the increase of temperature leads to the decrease of the vacuum. With the further increase of temperature, although the gas molecule has been desorbed, vacuum will still decrease as a result of the steam leakage from the soften rubber on the sealing surface of vacuum chamber. ${ }^{[6]}$ Therefore, the increase of temperature will cause the decrease of vacuum. However, as long as the temperature is not higher than $150^{\circ} \mathrm{C}$ and the vacuum still remain in $10^{-4} \mathrm{~Pa}$ level, the vacuum change caused by temperature will have no influence on nano-diamond field emission characteristics and the influence of temperature on vacuum can be ignored.

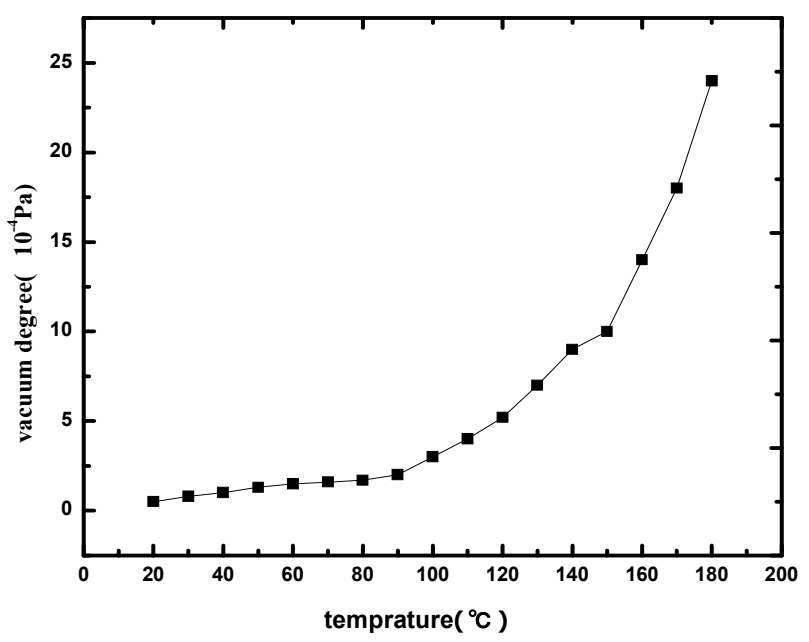

Figure 7. Curve of influence of temperature on vacuum

\section{CONCLUSION}

By the test experiment of field emission, the influence of temperature and vacuum on nano-diamond field emission characteristics was studied. The results show that temperature has a big influence on nano-diamond field emission. As the increase of temperature, field emission turn-on field decreases, and field emission current density increases. After the temperature rises to a certain degree, the increase of cathode thermal electron emission will submerge the field emission electron. The influence of thermal electron emission can be ignored at the temperature below $130{ }^{\circ} \mathrm{C}$. In low vacuum, scattering effect will make the field emission electron unstable. [7] However, when vacuum reaches above $10^{-4} \mathrm{~Pa}$ level, scattering has little influence on field emission electron, meaning that the cathode sample can emit the electron stably. Furnace temperature has certain influence on vacuum. With the rise of temperature, furnace vacuum will decrease. However, at the temperature below $150^{\circ} \mathrm{C}$, the change of vacuum caused by change of temperature will have no influence on nano-diamond field emission characteristics. ${ }^{[8]}$ Considered synthetically from the two aspects, the stable field emission conditions of nanodiamond are at the temperature below $130^{\circ} \mathrm{C}$ and in the vacuum above $10^{-4} \mathrm{~Pa}$ level.

\section{ACKNOWLEDGMENT}

We hereby express gratitude to our dear partner Yanyang Zhang, without his effort, this paper can not be accomplished. In the process of compilation, he made great contribution on test and data analysis.

\section{REFERENCES}

[1] C.A.Spindt, “A thin-film field-emission cathodes,".Appl.Phys, Maryland, vol.39, pp. 3504-3510, 1968

[2] Pimenov, S.M. Frolov, V.D. Kudryashov, A.V. Lamanov, M.M. Abanshin, N.P., etal. "Electron field emission from semiconducting nanowires", Diamond \&Related Materials,vol.17,pp.758-763, April/May 2008.

[3] Kang, W.P., Davidson, J.L., Howell, M., Bhuva, B., Kinser, D.L., Kerns, D.V., Li, Q.,Xu, J.F., “Micropatterned polycrystalline diamond field emitter vacuum diode arrays, ” J.Vac. Sci.Tech. vol. B14, pp. 2068-2071, May-Jun1996

[4] Yanning Yang, Zhiyong Zhang, Fuchun Zhang, Weihu Zhang and Junfeng Yan, "Temperature dependence of Field emission of nanodiamonds," Acta Phys. Sin., in press.

[5] Jung J H, Ju B K, Lee Y H, "Enhancement of electron emission efficiency and stability of molybdenum-tip field emitter array by diamond like carbon coating," Electron Device Letters, NJ,vol. 18, pp:197-199, May1997.

[6] Robertson J, "Electron Field Emission from Diamond and DiamondLike Carbon for Field Emission Displays,"Carbon, vol. 37, pp:759-763, April 1999

[7] Jian Chen, S. Z. Deng, Jun Chen, J. C. She, and N. S. Xu, "Preparation and characterization of nano structured film of graphitizeddiamond crystallites for field electron emission," Appl. Phys. Maryland, vol. 94, pp. 5429-5431, October 2003.

[8] W. G. Xie, Jian Chen, W. W. Ming, Jun Chen, J. Zhou, S. Z. Deng, and N. S. Xu, "Preparation and field emission property of nanodiamondcluster-embedded diamondlike carbon film” J.Vac. Sci. Tech., Vol. B26, pp. 1321-1325, Jul/Aug 2008 